\title{
Quark masses, the Dashen phase, and gauge field topology
}

\section{Michael Creutz}

Brookhaven National Laboratory, Upton, NY 11973, USA

\section{H I G H L I G H T S}

- The CP violating Dashen phase in QCD occurs when the up quark mass becomes sufficiently negative.

- Before reaching this phase, all physical hadronic masses and scattering amplitudes behave smoothly with the up-quark mass.

- The topological susceptibility of the gauge fields diverges to negative infinity as the Dashen phase is approached.

- A zero in the topological susceptibility provides a tentative signal for the point where the mass of the up quark vanishes.

- The universality of this definition remains unproven. Potential ambiguities are discussed.

\section{A R T I C L E I N F O}

\section{Article history:}

Received 20 August 2013

Accepted 3 October 2013

Available online 10 October 2013

\section{Keywords:}

Chiral symmetry

Lattice gauge theory

Gauge field topology

\begin{abstract}
A B S T R A C T
The CP violating Dashen phase in QCD is predicted by chiral perturbation theory to occur when the up-down quark mass difference becomes sufficiently large at fixed down-quark mass. Before reaching this phase, all physical hadronic masses and scattering amplitudes are expected to behave smoothly with the up-quark mass, even as this mass passes through zero. In Euclidean space, the topological susceptibility of the gauge fields is positive at positive quark masses but diverges to negative infinity as the Dashen phase is approached. A zero in this susceptibility provides a tentative signal for the point where the mass of the up quark vanishes. I discuss potential ambiguities with this determination.
\end{abstract}

(c) 2013 Elsevier Inc. All rights reserved.

E-mail address: creutz@bnl.gov. 


\section{Introduction}

Because of confinement, properties of quarks such as their masses cannot be observed directly. Indeed, what does a quark mass mean when free quarks do not exist? The commonly advocated approach is to do a lattice calculation and adjust the bare quark masses to match physical hadron properties. As one takes the continuum limit, the bare quark masses will flow as determined by the renormalization group. Because of asymptotic freedom and given a renormalization scheme, this behavior is precisely determined and allows us to extract a renormalized quark mass. This procedure is briefly reviewed in Appendix A. However, the details can depend on the precise lattice formulation, which raises the possibility of an ambiguity in defining the massless quark limit.

In a letter a number of years ago I pointed out that non-perturbative effects can leave an additive ambiguity in the definition of a non-degenerate quark mass [1]. This additive effect goes away in the isospin limit, a limit in which most lattice calculations are currently done. In the light quark regime, the quark masses are closely tied to the pseudo-scalar meson spectrum, with massless pions appearing as the up and down quarks become massless together.

Of course isospin in nature is only an approximate symmetry, being broken by the non-degeneracy of the up and down quarks as well as by electromagnetism. Indeed, it is somewhat remarkable that both effects are of comparable order to the hadron spectrum. The charged and neutral pion mass difference of a few $\mathrm{MeV}$ is generally regarded as being primarily from electromagnetic effects while the neutron-proton mass difference receives comparable contributions both from an underlying quark mass difference and from electromagnetism.

In chiral perturbation theory, to lowest order the pions have a mass squared proportional to the average of the up and down-quark masses. With isospin breaking, the neutral and charged pions are no longer exactly degenerate. The dominant effect is the energy in the electromagnetic field of the charged pion, leaving the neutral pion the lightest of the three. In addition, chiral perturbation theory predicts a small further splitting proportional to the square of the up-down mass difference [2]. Here I will concentrate on the quark mass difference effects, although presumably the electromagnetic effects have similar consequences and do not modify the qualitative picture below.

Since the pion mass is tied to the average of the up and down quark masses, there remains a gap in the hadronic spectrum if the down quark remains massive while the up-quark mass is taken to zero. Indeed, all physical processes are expected to behave smoothly in this limit. This brings us back to the question in Ref. [1] of whether there is a precise meaning to having a vanishing up-quark mass.

In this paper I discuss the qualitative behavior of the theory in the vicinity of vanishing up-quark mass, with particular attention to topological issues with the gauge fields. Topology is important since the pion mass difference receives a non-perturbative contribution through the induced mixing of the pion and the eta prime mesons, and the eta prime mass is dominated by non-perturbative effects from topology [3]. This mixing behaves smoothly even at a zero up-quark mass, despite the classical suppression of topological effects from a zero in the fermion determinant at non-trivial topology. In particular the eta prime mass, which comes primarily from these effects, remains of order the strong interaction scale throughout this region.

Continuing to a negative up-quark mass, I argue that the topological susceptibility will diverge to negative infinity as one approaches what is known as the Dashen phase [4]. Since this susceptibility is a positive quantity for positive quark masses, it must show a zero before this divergence becomes dominant. Assuming a single zero, this provides a natural definition of the point of vanishing up-quark mass. Nevertheless, since typical configurations in a path integral are non-differentiable, there may be subtleties that can lead to ambiguities in defining topology.

In Section 2 I review the standard picture from chiral perturbation theory of how the three pions are degenerate up to second order in the quark masses. In Section 3 I discuss the continuation to negative up-quark masses and why the Dashen phase is expected to appear. Section 4 discusses the topological susceptibility as a signal of where the up-quark mass vanishes. Here I argue for the divergence of this quantity as the Dashen phase is approached. Then in Section 5 I discuss the ambiguities that can arise in trying to define topology on the lattice. Section 6 discusses how these ambiguities are not resolved by current algebra and the symmetries of the theory. A brief summary of the conclusions appears in Section 7. 
A couple of closely related topics are relegated to appendices. Appendix A reviews how the bare quark mass always flows to zero in the continuum limit and how to define a renormalized mass from this flow. Appendix B discusses how the absence of a CP violating Theta parameter dependence of QCD at a vanishing mass is a tautology associated with using singular coordinates.

\section{The pseudo-scalar spectrum and the anomaly}

In this section I review the standard picture of the pseudo-scalar meson spectrum with two flavors of light fermions, the $u$ and $d$ quarks. From the fermion fields I can construct four pseudo-scalar operators

$$
\begin{aligned}
& \bar{u} \gamma_{5} d \sim \pi_{+} \\
& \bar{d} \gamma_{5} u \sim \pi_{-} \\
& \bar{u} \gamma_{5} u \\
& \bar{d} \gamma_{5} d .
\end{aligned}
$$

The first two create the charged pions, which have a natural mass controlled by the average of the up and down quark masses.

All of these operators involve a helicity flip. Helicity conservation is usually taken as a property of gauge theories coupled to light fermions. Thus for light quarks one might naively argue that the last two operators above would not mix significantly as that would involve helicity change for both the up and down quarks. This suggests that there would be two light neutral pseudo-scalars, one whose mass is controlled by the up-quark mass and a second by the down-quark mass.

Of course this expectation is naive. The anomaly induces a strong mixing between the neutral pseudo-scalar states. The symmetric combination

$$
\bar{u} \gamma_{5} u+\bar{d} \gamma_{5} d \sim \eta^{\prime}
$$

gains a mass of order $\Lambda_{q c d}$ and becomes the eta prime meson. How this mixing takes place through gauge configurations of non-trivial topology was elegantly described by 't Hooft [3], and the mixing term is often referred to as the 't Hooft vertex.

After the above mixing takes place, one is left with the orthogonal combination

$$
\bar{u} \gamma_{5} u-\bar{d} \gamma_{5} d \sim \pi_{0}
$$

to represents the neutral pion. Having approximately equal contributions from each quark, $\pi_{0}$ also has its mass dominantly controlled by the average quark mass. In the isospin limit of equal up and down quark masses, the three pions are degenerate up to electromagnetic splittings, which I ignore here.

Away from the isospin limit, small mixings of the eta prime and the neutral pion should remain. This allows for a purely hadronic contribution to the pion mass splitting proportional to $\left(m_{d}-m_{u}\right)^{2}$. The sign of this term is generally expected to drive the neutral pion mass down, although symmetry alone does not determine this. The qualitative picture is sketched in Fig. 1.

\section{The Dashen phase}

An important observation is that when the up-quark mass vanishes with a non-vanishing downquark mass, the theory retains a mass gap. This leads to the question of what happens as the up-quark mass is varied to negative values. The quark mass itself is only a formal parameter while the lightest physical particles are the pions. Their masses are controlled primarily by the average of the up and down quark masses, and it is natural to expect them to continue to drop until the up-quark mass is comparable in magnitude to the negative of the down-quark mass. The behavior of all physical quantities should be smooth in the quark masses in the immediate vicinity of vanishing up-quark mass as long as the down-quark mass remains non-zero. 


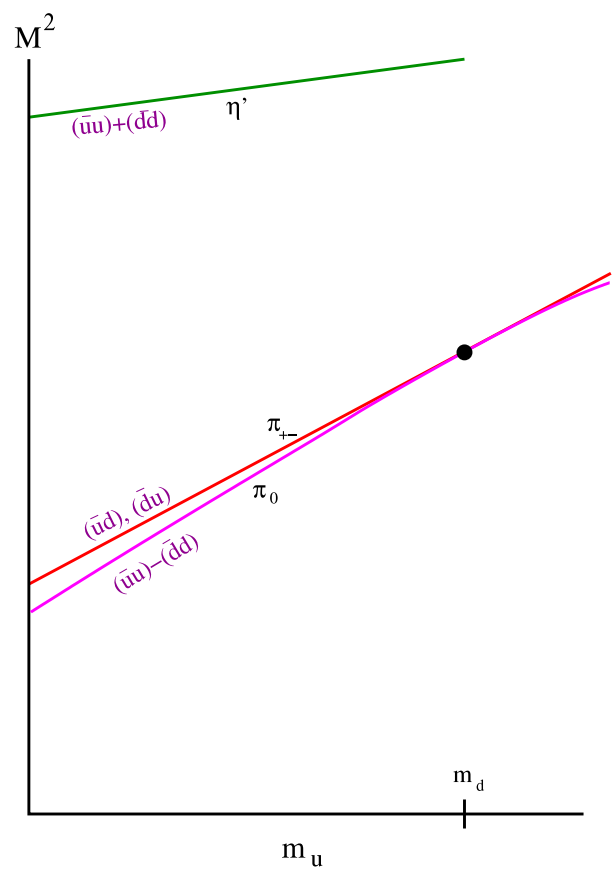

Fig. 1. The qualitative behavior of the meson spectrum as a function of the up-quark mass with a fixed non-vanishing downquark mass. Note that a mass gap persists as the up-quark mass approaches zero. Away from the isospin limit, mixing of the neutral pion with the eta prime can result in splitting between the neutral and charged pions. This splitting is quadratic in the quark mass difference.

On continuing to decrease the up-quark mass further into the negative region, the neutral pion mass will become progressively lighter and may eventually hit zero. Beyond such a point, the neutral pions should form a condensate with the pion field acquiring an expectation value. As the pion is $\mathrm{CP}$ odd, the new phase spontaneously breaks CP symmetry. The possibility of such a spontaneous breaking was postulated some time ago by Dashen [4]. As that was before the development of QCD as the theory underlying the strong interactions, his argument was based on current algebra ideas. The qualitative picture is illustrated in Fig. 2.

Note that this phenomenon is inherently non-perturbative. Naively the sign of a fermion mass is irrelevant in perturbative diagrams because it can be reversed by a chiral rotation. That rotation, however, is not valid due the chiral anomaly. This pion condensation occurs in a region where the product of the quark masses is negative. As is well known and discussed briefly in Appendix B, when the quark masses are made complex, the phase $\Theta$ of the product of the quark masses is a physical parameter [3]. Thus the Dashen phase occurs in a region where formally $\Theta=\pi$. But note from this example that there is also a finite region of negative up-quark mass before this condensation takes place. Thus whether or not there is a spontaneous breaking of $\mathrm{CP}$ at $\Theta=\pi$ will depend on the detailed values of the quark masses.

Vafa and Witten [5] have argued that QCD cannot spontaneously break CP. This argument assumed that one is working in a region where the fermion determinant appearing in the path integral is strictly positive, i.e. at $\Theta=0$. The Dashen phase is not a counterexample to their argument since it occurs where on some gauge field configurations the up-quark determinant is negative.

\section{Implications for the path integral}

The above discussion of how the physical states behave as the quark masses are varied implicitly applies to the properties of the Minkowski space theory. The picture raises some interesting questions 


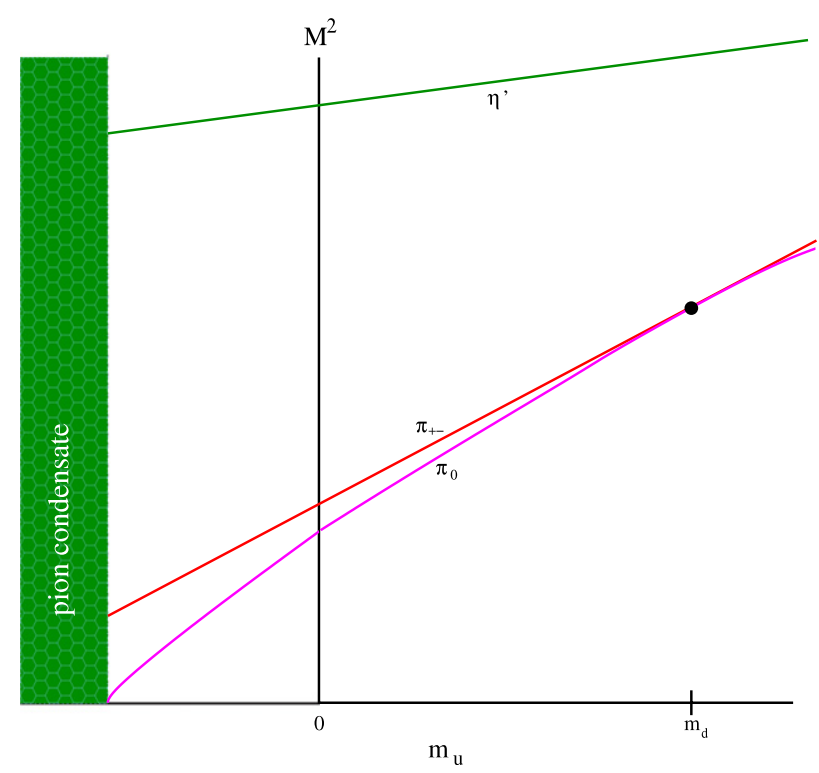

Fig. 2. Continuing the up-quark mass into the negative regime brings up the possibility of a vanishing pion mass and a subsequent condensation of the neutral pion field.

on how this physics appears in the path integral formulation. In particular, when fermions are integrated out in terms of the determinant of the Dirac operator, what are the implications for the behavior of this determinant?

Simple chiral Lagrangian arguments suggest that the boundary of the Dashen phase involves a second order phase transition [6,7]. At the critical point, the divergent correlation length is associated with the vanishing of the neutral pion mass. This example shows explicitly that one can have vanishing particle masses or divergent correlations in QCD at a point where none of the quark masses vanish. Because of the mass term, the Dirac operator will not have any small or vanishing eigenvalues.

In contrast, the fact that a mass gap persists in the vicinity of vanishing up-quark mass is an explicit example of a situation where the Dirac operator could have small eigenvalues but this does not imply important long range physics. Furthermore, the absence of any obvious structure raises the question of whether there is some physical way to determine exactly where the up-quark mass vanishes. That was the question raised in [1].

In the path integral approach, one standard answer to defining a vanishing up-quark mass is that this represents the point where the topological susceptibility vanishes. This is connected to the index theorem, which shows that the massless Dirac operator has a zero eigenvalue whenever the gauge field has non-trivial topology.

In any given gauge configuration the topological charge is formally defined as

$$
v=\frac{g^{2}}{16 \pi^{2}} \int d^{4} x F_{\mu \nu}(x) \tilde{F}_{\mu \nu}(x) .
$$

The susceptibility then follows from the path integral as

$$
\xi=\frac{1}{V}\left\langle v^{2}\right\rangle
$$

where $V$ is the space-time volume and the expectation is over the space of gauge configurations appropriately weighted with the action, including the fermion determinant. Using translational 
invariance, this can be written

$$
\xi=\left(\frac{g^{2}}{16 \pi^{2}}\right)^{2}\left\langle\int d^{4} x F_{\mu \nu}(x) \tilde{F}_{\mu \nu}(x) F_{\mu \nu}(0) \tilde{F}_{\mu \nu}(0)\right\rangle .
$$

For convenience below I abbreviate $F_{\mu \nu}(x) \tilde{F}_{\mu \nu}(x)$ as $F \tilde{F}(x)$.

It is important to note that since $F \tilde{F}$ is an odd operator under time reversal, the correlator

$$
\langle F \tilde{F}(x) F \tilde{F}(0)\rangle
$$

is negative at non-zero separations [8]. This is true even at positive quark masses where the path integral weight is strictly positive. Since the full susceptibility $\xi$ is defined as a square, it is expected to be positive when the quark masses are positive. The interpretation is that the correlator in Eq. (7) has a singular contact term providing a positive contribution that exceeds the negative part from nonzero separation. We will shortly see that the situation is more complicated when the up-quark mass is negative.

It is straightforward to show that as a function of the up-quark mass the topological susceptibility must vanish somewhere before reaching the Dashen phase. At negative quark mass the susceptibility, despite being formally a square, need not be positive. This is possible because the fermion determinant that enters the weight for the path integral can be negative. Although the path integral then loses its probability interpretation, presumably it still exists as a correct approach to the quantum theory.

For a negative up-quark mass near zero one expects higher topology configurations to be strongly suppressed. In this situation, the topological susceptibility should be dominated by the minimal nontrivial winding number of unity (winding number zero gives no contribution to the susceptibility), and in this case the fermion determinant is negative.

Not only can $\xi$ be negative, but as one approaches the endpoint of the Dashen phase, the topological susceptibility actually diverges to negative infinity. Since for positive mass the susceptibility is a positive definite quantity, there must be a zero somewhere before we reach the Dashen phase. The position of this zero is a natural definition of theory with zero up-quark mass.

The divergence of the susceptibility at the boundary of the Dashen phase is a direct consequence of the vanishing of the neutral pion mass at that point. Since isospin is broken, one expects some mixing between the neutral pion and both the eta prime meson and any pseudo-scalar glueball states. In particular, the operator $F \tilde{F}$ should have a finite amplitude to create the neutral pion; i.e. $\left\langle\pi_{0}|F \tilde{F}| 0\right\rangle \neq 0$. This means that at long distances the integral in Eq. (6) will diverge as $-1 / M_{\pi_{0}}^{2}$ due to pion intermediate states. Again, since $F \tilde{F}$ is odd under time reversal, this divergence is to negative infinity [8]. And since the boundary of the Dashen phase is determined by long distance pion dynamics, one does not expect the contact term to have a corresponding divergence, nor does one expect the coupling of $F \tilde{F}$ to the pion to vanish.

\section{Topology and the lattice}

So we see that as the up-quark mass varies, there must be a point where the topological susceptibility vanishes. But is this actually a physical concept, or could it depend on how the theory is regulated? This is a non-trivial point since in a path integral typical gauge configurations involve non-differentiable fields. Since gauge field topology is a non-perturbative concept, one must consider this question in the context of a non-perturbative regulator, i.e. the lattice. Indeed, numerous lattice schemes for defining topology have been proposed, but all presented so far leave room for ambiguities. This is actually a rather old topic; some numerical studies appear in Refs. [9-11].

One could just take a simple discretization of $F \tilde{F}$ and sum its value over configurations taken from a Monte Carlo simulation. One example of this is in Ref. [12]. The problem with this approach is that unlike with smooth continuum fields, any local definition of $F \tilde{F}$ will not in general be a total derivative and its integral will not be an integer. As such it is not a true topological object.

To get around this one might impose a smoothness condition on the lattice fields such that one can uniquely construct continuum field interpolating between the lattice sites $[13,14]$. This leaves open a 
few questions. First, a constraint on the lattice action that is not analytic in the gauge field variables will lead to a non-positive transfer matrix and correspondingly a non-Hermitean Hamiltonian when the cutoff is in place [15]. Second, as one takes the continuum limit, it is unclear to what extent different smoothness conditions will give the same result.

As a variation on the previous, one can perform a smoothing on the gauge field until it settles into a state of well defined topology. One example is to use a differential flow with the Wilson gauge action as a potential $[16,17]$. This procedure, however, is non-unique. First, the result can depend on the cooling action used. The standard Wilson gauge action is one choice, but it is unclear why this should be the appropriate form when effects of dynamical quarks are present. Second, the winding number found can depend on how long one cools. Without some constraint on the local action, topological objects can shrink with cooling and "fall through the lattice".

This leads us to a class of prescriptions based on the index theorem relating zero modes of the Dirac operator to the gauge field topology [18]. The simplest is to count the small real eigenvalues of the Wilson Dirac operator. The ambiguity here lies in the definition of "small". If one takes all real eigenvalues and counts them with their chirality, one will always get zero. Presumably one should ignore the eigenvalues in the doubler region, but with dynamical fermions the border between physical and doubler modes becomes blurred. Note that the zero of the topological susceptibility must occur for the up-quark mass in a region where negative real eigenvalues can occur. This allows a cancellation of the purely positive contribution when there are no negative real eigenvalues against the negative contribution when such modes do exist.

An action satisfying the Ginsparg-Wilson relation [19] can have exact zero modes and they properly match with the gauge field index when the gauge fields are smooth. With such an approach, a vanishing up quark mass automatically suppresses all configurations of non-zero topology since the fermion determinant is zero for any such. This, however, does not resolve the issue because there are many operators that satisfy this relation, and for general fields they do not all give the same index. The Neuberger construction of the overlap operator [20] depends on a projection from a kernel such as the Wilson operator. The location of the projection point suffers the same ambiguity as in the previous paragraph. Indeed, one would expect the eigenvalue count for typical configurations to decrease as the projection point is lowered and fewer real eigenvalues of the Wilson kernel lie below this location. Again, the question here is tied to the real eigenvalues, which are inherently non-perturbative. It is often asserted that with the Wilson kernel there will be a region where the density of real eigenvalues will decrease rapidly enough to eliminate this ambiguity, although the numerical evidence for this remains limited [21-23].

I note in passing that the staggered fermion approach to lattice fermions retains the naive symmetry under reversing the sign of any quark mass. This ensures the uniqueness of the zero mass theory. However this approach suffers doubling issues, making any massless species actually multiply degenerate. The rooting procedure often advocated to circumvent this problem is known to be an approximation which severely mutilates the 't Hooft vertex [24,25]. Unlike as discussed in Section 2, taste non-singlet $\bar{u} \gamma_{5} u$ mesons will survive the procedure with masses controlled by the up-quark mass alone. Pairs of these spurious mesons will cause unphysical thresholds in scattering amplitudes when the physical quarks are not degenerate, even when all quarks have positive mass.

\section{Symmetries and currents}

One might try to circumvent these issues via continuum concepts using currents related to the symmetries of the theory. Indeed, Dashen's discussion of the possibility of a CP violating phase was based on current algebra and was presented before QCD became the accepted theory for the strong interactions. In this section I will discuss how the lack of any symmetry at vanishing up quark mass leaves open an ambiguity in defining this point.

When the quark mass difference vanishes we have isospin symmetry. This makes a zero mass difference a well defined concept. To get a handle on the quark mass difference as we move away from this point, one might consider the partial conservation of a charged vector current. For a small mass difference the divergence of such a current is proportional to the mass difference with a coefficient depending on the chiral condensate. However, as the mass difference grows, the behavior of this 
divergence ceases to be linear. In particular, as one passes through the boundary of the Dashen phase, an associated non-analyticity will appear in all physical quantities, including the charged currents. It is these non-linearities that leave room for the ambiguities discussed in the previous section.

Another interesting situation occurs when the sum of the up and down quark masses vanishes. Then, when the quark mass difference does not vanish, we are deep in the Dashen phase. Here we can move the sign of the negative quark mass into the gauge field topology with a term in the action proportional to $F \tilde{F}$. Then there is again an explicit isospin symmetry between the quarks. This serves to protect the mass sum from renormalization. We again have a symmetry that protects the average quark mass from an additive renormalization.

The issue of a vanishing up quark mass when the down quark is massive is not associated with either of these two symmetry points. Rather, it involves neither the sum nor the difference of the quark masses vanishing. In this case these quantities are not related by any fundamental symmetry. For example, they transform differently under isospin; the sum of the masses is an isoscalar quantity and the difference is an isovector. While both are individually multiplicatively renormalized, there is no rigorous symmetry that relates their non-perturbative renormalization factors.

A further complication is that non-perturbative physics requires a lattice approach, and then properly defined currents are dependent on the detailed fermion formulation. With all lattice actions, the conserved currents are not simple on-site quantities. Particularly with Ginsparg-Wilson type operators, they involve fields spread over formally unlimited distances. Given a particular lattice formulation, the underlying quark masses may be well defined, but there appears to be no fundamental reason to require the physics at a vanishing quark mass to be universal between schemes.

\section{Conclusions}

There are three main conclusions to draw from this discussion. First, the topological susceptibility must diverge as the Dashen phase is approached. Second, since this divergence is to negative infinity, with any sensible regulator there must exist a zero in the susceptibility as the up quark mass varies up to its physical value. Third, it is unclear whether the location of this zero is universal between lattice schemes.

The third point is somewhat controversial since this zero provides a tentative definition of the point where the up quark is massless. Because all known methods to locate this point in a lattice simulation appear to have some arbitrariness, it remains unclear whether the zero must scale in a manner to give a unique continuum limit for more physical quantities. In particular, two different lattice cutoffs taken to the continuum limit while forcing $m_{u}=0$ can potentially give different ratios for physical hadron masses.

From a phenomenological point of view, there seems to be little reason to care whether the upquark mass is ambiguous. This is not a directly observable quantity, and both hadron masses and scattering amplitudes behave smoothly as the quark mass passes through zero. For confidence in lattice results, it is important to compare physical results with different schemes. The issues raised here suggest that using quark masses or topological susceptibility directly for such matching might lead to unpredictable results.

\section{Acknowledgments}

This manuscript has been authored under contract number DE-AC02-98CH10886 with the U.S. Department of Energy. Accordingly, the U.S. Government retains a non-exclusive, royalty-free license to publish or reproduce the published form of this contribution, or allow others to do so, for U.S. Government purposes.

\section{Appendix A. Quark masses and the renormalization group}

In lattice gauge language, asymptotic freedom tells us how to vary the bare gauge coupling and quark masses to take the continuum limit. This variation is manifest via the "renormalization group 
equations"

$$
\begin{aligned}
& a \frac{d g}{d a}=\beta(g)=\beta_{0} g^{3}+\beta_{1} g^{5}+\cdots+\text { non-perturbative } \\
& a \frac{d m}{d a}=m \gamma(g)=m\left(\gamma_{0} g^{2}+\gamma_{1} g^{4}+\cdots\right)+\text { non-perturbative. }
\end{aligned}
$$

Here the three initial perturbative coefficients $\beta_{0}, \beta_{1}, \gamma_{0}$ are scheme independent and known [26-32]. These equations are easily integrated to show

$$
\begin{aligned}
& a=\frac{1}{\Lambda} e^{-1 / 2 \beta_{0} g^{2}} g^{-\beta_{1} / \beta_{0}^{2}}\left(1+O\left(g^{2}\right)\right) \\
& m=M g^{\gamma_{0} / \beta_{0}}\left(1+O\left(g^{2}\right)\right) .
\end{aligned}
$$

The quantities $\Lambda$ and $M$ are "integration constants" for the renormalization group equations. Rewriting these relations gives the coupling and mass flow in the continuum limit $a \rightarrow 0$

$$
\begin{aligned}
& g^{2} \sim \frac{1}{\log (1 / \Lambda a)} \rightarrow 0 \text { "asymptotic freedom" } \\
& m \sim M\left(\frac{1}{\log (1 / \Lambda a)}\right)^{\gamma_{0} / \beta_{0}} \rightarrow 0 .
\end{aligned}
$$

Here $\Lambda$ is usually regarded as the "QCD scale" and $M$ as the "renormalized quark mass".

Thus it would seem that given some renormalization scheme we have a way to define the quark mass. The issue with defining a vanishing quark mass is complicated due to the "non-perturbative" terms in Eq. (8). Because the integration constant $\Lambda$ introduces a new scale into the theory, the nonperturbative contribution to the mass flow need not be proportional to the bare mass. Without some symmetry to prevent it, an additive mass shift proportional to $\Lambda$ is generally possible. And with only one massless quark, the anomaly breaks the naive chiral symmetry that would prevent such a term.

Of course with multiple degenerate quarks we would have flavor non-singlet chiral symmetries that would remove any such additive renormalization. But in the case considered here, we are keeping the down-quark mass fixed at a non-zero value. The fact that one would recover chiral symmetry if the down-quark mass was also zero indicates that the size of any potential ambiguity in the up-quark mass should be proportional to the down-quark mass.

\section{Appendix B. The Theta parameter and CP symmetry}

As is well known, QCD as a renormalizable quantum field theory has the possibility of including a CP violating term, usually called $\Theta$. This is closely tied to the anomaly and can be moved around between appearing either in the quark mass matrix or the appearance of a topological term in the gauge field action. One often proposed way to locate the vanishing up-quark mass point is that at this point the theory is independent of the Theta parameter. But in the light of the above discussion this is actually a tautology.

After rotating the theta parameter into the up-quark mass, this mass term takes the form

$$
m_{1} \bar{\psi} \psi+i m_{5} \bar{\psi} \gamma_{5} \psi \text {. }
$$

From this, it is conventional to define a complex mass parameter

$$
m=m_{1}+i m_{5} .
$$

The phase of this quantity is the parameter $\Theta$. Of course, if $m$ vanishes as a complex number, $\Theta$ ill defined and irrelevant.

The problem with this interpretation is that $m_{1}$ and $m_{5}$ are independent parameters. And the above discussion suggested an ambiguity in defining where $m_{1}$ vanishes. This ambiguity feeds through into an ambiguity in defining $\Theta$. In a sense the conventional approach attempts to set up polar coordinates about a point which is not a natural origin. 


\section{References}

[1] Michael Creutz, Phys. Rev. Lett. 92 (2004) 162003.

[2] Michael Creutz, Phys. Rev. D 52 (1995) 2951-2959.

[3] Gerard 't Hooft, Phys. Rev. D 14 (1976) 3432-3450.

[4] Roger F. Dashen, Phys. Rev. D 3 (1971) 1879-1889.

[5] Cumrun Vafa, Edward Witten, Phys. Rev. Lett. 53 (1984) 535.

[6] Michael Creutz, Phys. Rev. Lett. 92 (2004) 201601.

[7] Michael Creutz, Phys. Rev. D 83 (2011) 016005.

[8] Erhard Seiler, Phys. Lett. B 525 (2002) 355-359.

[9] M. Teper, Phys. Lett. B 162 (1985) 357.

[10] Falk Bruckmann, Florian Gruber, Karl Jansen, Marina Marinkovic, Carsten Urbach, et al., Eur. Phys. J. A 43 (2010) $303-311$.

[11] Peter J. Moran, Derek B. Leinweber, Jianbo Zhang, Phys. Lett. B 695 (2011) 337-342.

[12] Michael Creutz, Ann. Physics 326 (2011) 911-925.

[13] M. Luscher, Comm. Math. Phys. 85 (1982) 39.

[14] David H. Adams, Ann. Physics 296 (2002) 131-151.

[15] Michael Creutz, Phys. Rev. D 70 (2004) 091501.

[16] R. Narayanan, H. Neuberger, J. High Energy Phys. 0603 (2006) 064.

[17] Martin Luscher, J. High Energy Phys. 1008 (2010) 071.

[18] David H. Adams, J. Math. Phys. 42 (2001) 5522-5533.

[19] Paul H. Ginsparg, Kenneth G. Wilson, Phys. Rev. D 25 (1982) 2649.

[20] Herbert Neuberger, Phys. Lett. B 417 (1998) 141-144.

[21] Robert G. Edwards, Urs M. Heller, Rajamani Narayanan, Phys. Rev. D 59 (1999) 094510.

[22] Leonardo Giusti, G.C. Rossi, M. Testa, Phys. Lett. B 587 (2004) 157-166.

[23] Martin Luscher, Filippo Palombi, J. High Energy Phys. 1009 (2010) 110.

[24] Michael Creutz, Phys. Lett. B 649 (2007) 230-234.

[25] Michael Creutz, Acta Phys. Slovaca 61 (2011) 1-127.

[26] H. David Politzer, Phys. Rev. Lett. 30 (1973) 1346-1349.

[27] D.J. Gross, Frank Wilczek, Phys. Rev. Lett. 30 (1973) 1343-1346.

[28] D.J. Gross, Frank Wilczek, Phys. Rev. D 8 (1973) 3633-3652.

[29] William E. Caswell, Phys. Rev. Lett. 33 (1974) 244.

[30] D.R.T. Jones, Nucl. Phys. B 75 (1974) 531.

[31] J.A.M. Vermaseren, S.A. Larin, T. van Ritbergen, Phys. Lett. B 405 (1997) 327-333.

[32] Konstantin G. Chetyrkin, Mikolaj Misiak, Manfred Munz, Nucl. Phys. B 518 (1998) 473-494. 